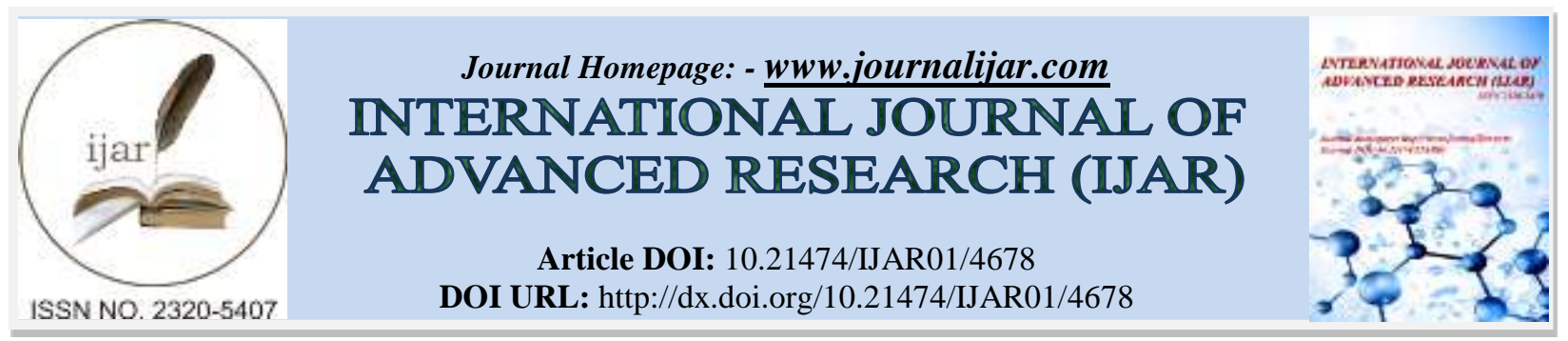

RESEARCH ARTICLE

\title{
CD160 EXPRESSION IN B CHRONIC LYMPHOCYTIC LEUKEMIA (B-CLL.)
}

Deena Elshabrawy ${ }^{1}$, Yasmin Elsakhawy ${ }^{2}$ and Eman Ibrahim ${ }^{3}$.

1. Assistant professor, Benha University, faculty of medicine.Egypt.

2. Assistant professor of Clinical Pathologyr Ain Shams University, Faculty of Medicine, Egypt.

3. M.B.B.Ch, Msc.

\section{Manuscript Info}

(.........................

Manuscript History

Received: 24 April 2017

Final Accepted: 27 May 2017

Published: June 2017

Key words:-

CD160, CLL, FLOWCYTOMETRY.

\section{Abstract}

CLL is usually diagnosed through the charachteristic morphology/immunophenotype of the lymphocytes, but some CLL cases remain atypical resulting in uncertain diagnosis. CD160 is a glycosylphosphatidylinositol-Linked receptor found on normal natural killer (NK) and T cells, but not B cells. Its activation protects against apoptosis and increase mean cell viability. The aim of the study is to analyze expression of CD160 in B-CLL and if there is relation with clinical and laboratory parameters. CD 160 expression was assessed using Flow Cytometry, in 60 newly diagnosed CLL patients in addition to 30, matched age and sex, normal subjects who served as a control group. We found that, CD160 was expressed on a greater percentage of B-CLL cells in patients than in control group ( $p<0.01)$ with very high sensitivity $(96.7 \%)$ and specificity $(100 \%)$, so it can represent a useful diagnostic tool in B-CLL cases. There was no significant difference of CD160 expression in various stages in Binet and Rai staging. We demonstrated a significant statistical positive correlation between CD160 and CD20 ( $<<0.05$ ) and a highly significant statistical positive correlation between CD160 and CD19 and CD38 ( $p<0.01$ ). No significant correlation was found between CD160 and CD23, CD79b, SIgM, FMC7, CD5 and CD10.There was no significant statistical correlation between CD160 and Hemoglobin level(Hb), platelet count(PLT), total leukocytic count(TLC), Absolute and relative lymphocytic count, bone marrow lymphocytes (BML) and Lactate dehydrogenase(LDH). We concluded that; CD160 was expressed on a greater percentage of B-CLL cells in patients than in control group with very high sensitivity and specificity, so it can represent a useful diagnostic tool in B-CLL cases. CD160 expression may be useful prognostic marker in patients with B- CLL.

Copy Right, IJAR, 2017,. All rights reserved.

\section{Introduction:-}

CD160 is a glycophosphatidylinositol (GPI)- linked membrane protein, it recruits phosphoinositide-3 kinase (PI3K) and induces phosphorylation of Akt and extracellular signal- related kinase downstream signaling elements in NK cells. In CD8+ T cells, CD160 enhances CD3 induced proliferation and improves CD3 induced cytotoxicity (1). 
In healthy people, CD160 distribution is restricted to both circulating and tissue cytotoxic NK cells as well as T cells, with no expression in normal B cells. In disease state, CD160 is up regulated on CD3+ CD8+ T cells during infection with human immunodeficiency virus and is expressed on neoangiogenic vasculature of tumors as well as CD4+ T cells infiltrating inflammatory skin diseases (2).

Although CLL cells are characterized by being largely in G0/G1 phase of the cell cycle and long lived with high levels of antiapoptotic molecules in vivo, they rapidly die when cultured in vitro (3). However, some cytokines, chemokines, albumin, and stromal cells have been shown to protect from spontaneous apoptosis in vitro (4).

Constitutive activation of Akt was shown to increase expression of antiapoptotic proteins Mcl-1 and Bcl-xl and cell survival in CLL. CD160 promotes CLL survival by up-regulating Bcl-2, Bcl-xL, and Mcl-1 expression and blocking cytochrome c release, collapse, and caspase activation (5).

Antiapoptotic Bcl-2, Bcl-xL, and Mcl-1 are localized in the inner and/or outer mitochondrial membranes and prevent apoptosis by blocking cytochrome $\mathrm{c}$ release from the outer mitochondrial membrane and inhibiting the collapse induced by Bax activation (6).

Caspase activation is a crucial step for apoptosis in both the mitochondrial-dependent and -independent pathways. Extrinsic death factors, such as TNF-alpha and TNF-related apoptosis-inducing ligand, activate caspase-8, which then triggers the apoptotic process by directly activating downstream caspase- 3 to induce cytochrome $\mathrm{c}$ release, which initiates caspase-9 activation, which in turn activates caspase-3, but not caspase 8. CD160 activation decreased not only caspase- 3 and caspase- 9 activity, but also caspase- 8 activity, indicating that spontaneous apoptosis of CLL cells in vitro may involve both mitochondrial-dependent and independent pathways (6).

Also, CD160 can regulate the shift of quiescent CLL cells into the cell cycle and an increase in S phase distribution. In vitro, many cytokines have pro survival and antiapoptotic function in CLL. Activation of CD160 triggered very high-level IL- 6 and IL-8 secretion, with CD160 mediating a 50-fold increase in IL-6 and 15-fold in IL-8 which provides a survival signal in CLL leading to Bcl-2 up-regulation. Survival and activation signals mediated by CD160 showed dose-dependent suppression by phosphoinositide-3 kinase (PI3K) inhibitors. Thus, in vitro, CLL cells can use the CD160 pathway for survival and activation, mimicking CD160 signaling in normal NK and CD8 T cells. Establishing the pathophysiologic relevance of these findings may reveal new therapeutic targets. (7).

The aim of the study is to analyze expression of CD160 in B-CLL and if there is relation with clinical and laboratory parameters. CD 160 expression was assessed using Flow Cytometry, in 60 newly diagnosed CLL patients in addition to 30, matched age and sex, normal subjects who served as a control group. We found that, CD160 was expressed on a greater percentage of B-CLL cells in patients than in control group with very high sensitivity and specificity, so it can represent a useful diagnostic tool in B-CLL cases.

\section{Patients And Methods:-}

The present study was conducted on 60 newly diagnosed adult patients with chronic lymphocytic leukemia. The patients were recruited for the study based on standard clinical, hematological and immunophenotypic criteria for diagnosis of B-CLL. The patients were assessed at their first presentation to the Hematology/Oncology Clinic, Ain Shams University Hospital. Their ages ranged from 45 to 80 years with a mean of $59.7 \pm 8.52$ year. Thirty age and sex matched hematologically normal individuals were included and served as a control group.

Measuring expression of CD160 in patients and CD160 /CD19 in control group was done by using flow cytometry. Whole blood samples were analyzed within 24 hours. Leukocytes $\left(1 \times 10^{6}\right)$ were labeled with the appropriate antibody for 15 minutes at room temperature in darkness. Erythrocyte lysis was performed using Pharm Lyse (Becton Dickinson), a buffered ammonium chloride-based lysing solution, according to the manufacturer's recommendations, then washed in BD Cell Wash (BD Biosciences). A minimum of 10000 lymphocyte-gated events was acquired for each patient Positivity was defined as $>20 \%$ of leukemic cells expressing a given surface antigen. A sequential gating strategy for the specific identification of CD160 on CD5+CD19+ B cells using multicolor flow cytometry was established using Coulter EPICS-XL, USA. 


\section{Statistical Analysis:-}

Data were collected, verified, revised then edited on personal computer(PC). Then data were analyzed using Statistical package for Social Science (SPSS).

- Chi-Square test $\left(\mathrm{x}^{2}\right.$-value) was used for comparing qualitative data.

- Independent test (t-value) was used for comparing quantitative data.

- Pearson-correlation co-efficient(r) was used in correlation studies.

- A receiver operating characteristic (ROC) curve was applied to assess the diagnostic performance of CD160 in CLL patients versus the healthy control group at a cut off level 19.9.

\section{Results:-}

Our study conducted on 60 newly diagnosed CLL patients. According to Rai staging, there were; $2(3.3 \%)$ patient in stage $0,10(16.7 \%)$ patients in stage I, $14(23.3 \%)$ patients in stage II, 22 (36.7) patients in stage III, $12(20 \%)$ patients in stage IV. According to Binet staging; 18 (30\%) were in stage A, $22(36.7 \%)$ patients were in stage B and $20(33.3 \%)$ patients were in stage C. CD 160 expression was measured in patient and control group. We found that CD 160 was expressed in 58/60(96.7\%) CLL patients, while no CD 160 expression was detected in control group (table 1). Using the ROC curve to evaluate the sensitivity and specificity of CD 160 as a diagnostic marker for CLL (Table 2) (Figure1), we found that CD 160 expression has high sensitivity (96.7\%) and high specificity (100\%). No significant correlation was found between CD 160 expression and age of the patients or patient's clinical findings including lymphadenopathy, splenomegaly or hepatomegaly. Our result showed no correlation between CD 160 expression and laboratory findings including Hb, PLT, TLC, lymphocytic \%, ALC, BML, LDH (P value >0.05).

There was no significant statistical correlation between CD160 and CD23, CD79, SIGM, FMC7, CD5 and CD10 (P value >0.05). There was a significant statistical positive correlation between $\mathrm{CD} 160$ and $\mathrm{CD} 20(\mathrm{P}$ value $=$ 0.012). and a Highly significant statistical positive correlation between CD160 and CD19 and CD38, $(\mathrm{P}$ value $=$ 0.001 and 0.006) respectively (table3). Regarding CLL staging no correlation was found between CD 160 expression and both Rai\& Binet stagin.

\section{ROC Curve}

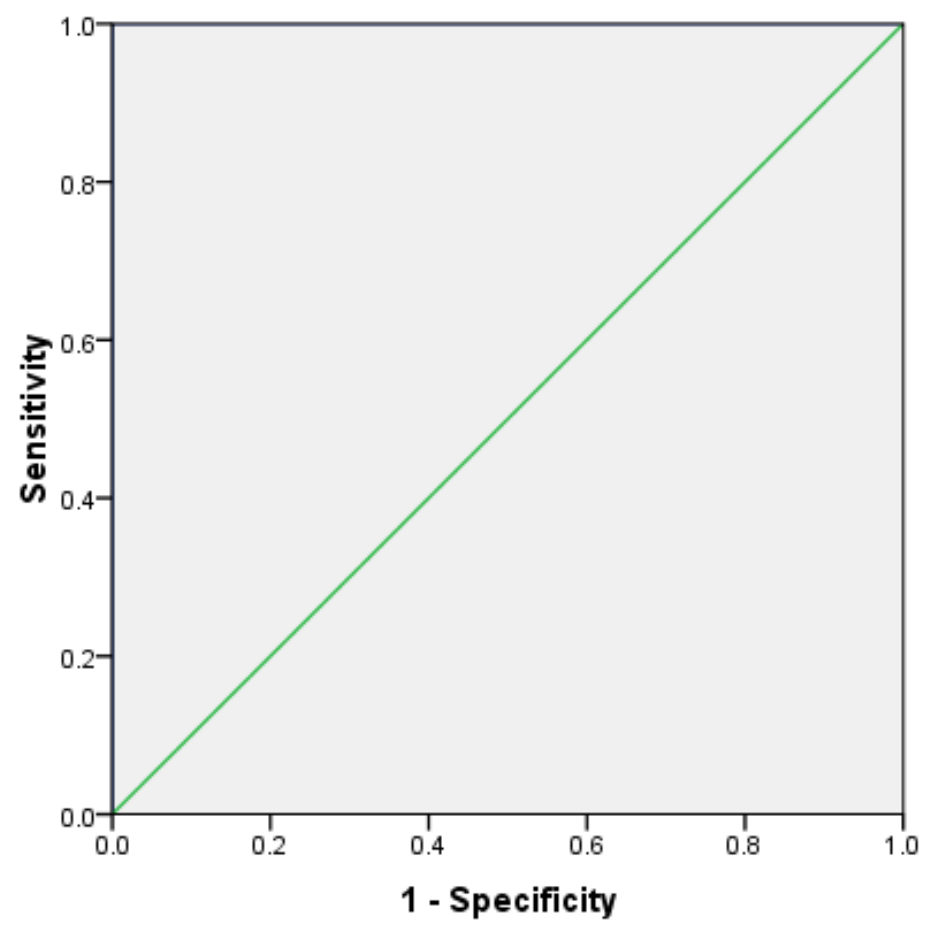

Fig. 1:- Sensitivity and specificity of CD160 in diagnosis of CLL. 
Table 1:- CD160 expression in patients and control.

\begin{tabular}{|c|c|c|c|c|c|c|}
\hline \multirow[t]{2}{*}{ CD 160} & \multicolumn{2}{|c|}{ CLL cases } & \multicolumn{2}{|c|}{ Control group } & \multirow{5}{*}{$\begin{array}{c}\text { x2 test } \\
36.67\end{array}$} & \multirow{5}{*}{$\begin{array}{c}\text { P value } \\
0.001 \mathrm{HS}\end{array}$} \\
\hline & No & $\%$ & No & $\%$ & & \\
\hline Positive & 58 & 96.7 & 0 & 0.0 & & \\
\hline Negative & 2 & 3.3 & 30 & 100 & & \\
\hline Total & 60 & 100 & 30 & 100 & & \\
\hline
\end{tabular}

Table 2:- Sensitivity and specificity of CD160 expression using ROC curve.

\begin{tabular}{|c|c|c|c|c|c|c|c|}
\hline CD160\% & $\begin{array}{c}\text { AUC area } \\
\text { under } \\
\text { curve. }\end{array}$ & $\begin{array}{c}\text { The best cut } \\
\text { off point }\end{array}$ & Sensitivity & Specificity & $\begin{array}{c}\text { PPV positive } \\
\text { predictive } \\
\text { value }\end{array}$ & $\begin{array}{c}\text { NPV negative } \\
\text { predictive } \\
\text { value }\end{array}$ & \begin{tabular}{c} 
Accuracy \\
\cline { 2 - 7 }
\end{tabular} \\
\hline 1.0 & 19.9 & $96.7 \%$ & $100 \%$ & $100 \%$ & $93.8 \%$ & $97.8 \%$ \\
\hline
\end{tabular}

Table 3:- Correlation between CD160 expression and other CD markers expression in CLL patients.

\begin{tabular}{|c|c|c|c|c|}
\hline \multicolumn{2}{|c|}{ CLL Case group } & Pearson correlation & P value & Significance \\
\hline \multirow{4}{*}{ CD160\% } & CD19 \% & 0.461 & 0.01 & HS \\
\cline { 2 - 5 } & CD20 \% & 0.451 & 0.012 & S \\
\cline { 2 - 5 } & CD23 \% & 0.188 & 0.320 & NS \\
\cline { 2 - 5 } & CD79b \% & 0.242 & 0.198 & NS \\
\cline { 2 - 5 } & SIGM \% & 0.005 & 0.978 & NS \\
\cline { 2 - 5 } & FMC7 \% & 0.351 & 0.057 & NS \\
\cline { 2 - 5 } & CD5 \% & -0.032 & 0.823 & NS \\
\cline { 2 - 5 } & CD10 \% & 0.043 & 0.006 & HS \\
\cline { 2 - 5 } & CD38 \% & 0.490 & & \\
\hline
\end{tabular}

$\mathrm{P}$ value $\geq 0.05 \rightarrow$ non-significant $(\mathrm{NS})$.

$\mathrm{P}$ value $<0.05 \rightarrow$ significant $(\mathrm{S})$.

$\mathrm{P}$ value $<0.01 \rightarrow$ highly-significant $(\mathrm{HS})$.

Table 4:- CD160 expression and Rai staging in CLL patients

\begin{tabular}{|c|c|c|c|c|c|}
\hline CLL GROUP & CD160\% & Mean \pm SD & Range & Test of ANOVA (F test) & P value \\
\hline \multirow{3}{*}{ Rai Staging } & 0 & $43.0 \pm-$ & $43-43$ & & \\
\cline { 2 - 4 } & I & $53.84 \pm 34.49$ & $15.8-89.7$ & \multirow{2}{*}{$0.855(\mathrm{NS})$} \\
\cline { 2 - 4 } & II & $63.23 \pm 15.11$ & $47.1-92$ & 0.33 & \\
\cline { 2 - 4 } & III & $64.09 \pm 26.79$ & $24.2-95.1$ & & \\
\cline { 2 - 4 } & IV & $56.95 \pm 18.24$ & $30.9-84.7$ & & \\
\end{tabular}

Table 5:- CD160 expression and Binet staging in CLL patients

\begin{tabular}{|l|l|l|l|c|l|}
\hline CLL GROUP & CD160\% & Mean \pm SD & Range & Test of ANOVA (F test) & P value \\
\hline \multirow{3}{*}{ Binet Staging } & A & $57.26 \pm 28.23$ & $15.8-92$ & & \\
\cline { 2 - 4 } & B & $60.31 \pm 20.67$ & $28.5-95.1$ & & \multirow{2}{*}{0.104} \\
\cline { 2 - 4 } & C & $62.28 \pm 23.55$ & $24.2-90.3$ & $0.901(\mathrm{NS})$ \\
\hline
\end{tabular}

\section{Discussion:-}

B-cell chronic lymphocytic leukemia (CLL) is characterized by the expansion of monoclonal CD5 B lymphocytes that accumulate in peripheral blood (PB), secondary lymphoid organs, and bone marrow. CLL remains incurable and this is partly attributable to cells being in the G0/G1 phase of the cell cycle and having high levels of antiapoptotic Bcl-2 family proteins which regulate mitochondrial outer membrane permeability and cytochrome $\mathrm{c}$ release from the mitochondrial inter membrane space (3).

These malignant cells exhibit genetic abnormalities that can modify their resistance to apoptosis and response to selected microenvironmental signals giving both a growth and survival advantage. Despite their prolonged survival in vivo, CLL cells rapidly undergo spontaneous apoptosis once removed from their microenvironment, suggesting that survival signals available in vivo have been lost in culture condition. This spontaneous in vitro apoptosis can be prevented by some cytokines, albumin and stromal cells (8). 
CD160 is a glycosylphosphatidylinositol-Linked receptor found on normal natural killer (NK) and T cells, but not B cells. Its activation protects against apoptosis and increase mean cell viability. This was associated with upregulation of $\mathrm{Bcl}-2$, Bcl-xL, and $\mathrm{Mcl}-1$, leading to increase cell survival (5). CD160 triggering reduced mitochondrial membrane potential collapse and cytochrome c release. Its stimulation also induced DNA synthesis, cell cycle progression and proliferation, and its signaling led to CLL cells secretion of IL-6 and IL-8 (9).

To highlight the role of CD160 expression on B-CLL cells, this study was conducted to evaluate the clinical significance of CD160 expression on B-CLL cells and its relationship with the clinical and laboratory parameters. To achieve this aim, the current study included 60 newly diagnosed B-CLL patients in addition to 30 age and sex matched apparently normal individuals as a control group.

In this study we found that, patients with CLL had significant higher levels of CD160 than healthy controls. This was consistent with what was reported by other study $((10)$. This can be explained by the effect of CD160 which promotes CLL survival by up regulation of BCL2, BCLx and MCL expression and blocking cytochrome c release and caspase activation (7).

In the current study according to ROC curve, sensitivity of CD160 was $96.7 \%$, This sensitivity is simillar to what reported by Farren et al,2011(98\%). In the present study, the specificity was $100 \%$. In addition, positive and negative predictive value (PPV and NPV) of CD160 were 100\%, 93.8\% respectively. This high sensitivity and specificity of CD160, in addition to its relatively simple evaluation, as CD160 is surface marker that can be easily evaluated using flow cytometry, may serve it as a diagnostic marker in patients with CLL.

There was no significant statistical correlation between CD160 and Hb, PLT, TLC, lymphocytic\%, ALC, BML and $\mathrm{LDH}$.

Also, there was no significant statistical correlation between CD160 and CD23, CD79b, SIGM, FMC7, CD5 and CD10, But There was a significant statistical positive correlation between CD160 and CD20 and a highly significant statistical positive correlation between CD160 and CD19 and CD38.

As the present study demonsteratd a highly statistically significant positive correlation between CD160 and CD38 which was considered as valuable prognostic marker (11). This finding suggests that, CD160 has a role in prognosis. However, there was no significant difference of CD160 expression in various stages in Binet and Rai staging. we concluded that; CD160 was expressed on a greater percentage of B-CLL cells in patients than in control group with very high sensitivity and specificity, so it can represent a useful diagnostic tool in B-CLL cases.CD160 expression may be useful prognostic marker in patients with B- CLL.

Disclosure of conflicts of interest:- We declare that we have no conflicts of interest to disclose.

Ethical Approval:- "All procedures performed in studies involving human participants were in accordance with the ethical standards of the Benha university Faculty of medicine research committee and with the 1964 Helsinki declaration and its later amendments."

Informed Consent:- "Informed consent was obtained from all individual participants included in the study."

\section{References:-}

1. Rabot M, Elcosta H, Polgar B, Cardine A M, Aguerre-Girr Aliz M, Salvatore V, Bensussan A Le Bouteiller Ph: CD160-activating NK cell effector functions depend on the phosphatidylinositol 3-kinase recruitment. Int Immunol (2007) 19 (4): 401-409.

2. Abecassis S1, Giustiniani J, Meyer N,$\underline{\text { Schiavon } \mathrm{V}}$, Ortonne N , Campillo JA, Bagot M, Bensussan A: Identification of a novel CD160+ CD4+ T-lymphocyte subset in the skin: a possible role for CD160 in skin inflammation. J Invest Dermatol. 2007 May;127(5):1161-6.

3. Reed J: CBcl-2-family proteins and hematologic malignancies: history and future prospect.Blood (2008) ;111(7):3322.

4. Niedermeier M, Hennessy BT and Knight ZA: Isoform-selective phosphoinositide 3_-kinase inhibitors inhibit CXCR4 signaling and overcome stromal cell-mediated drug resistance in chronic lymphocytic leukemia: a novel therapeutic approach. Blood (2009);113(22):5549. 
5. Balakrishnan K, Burger JA, Wierda WG and GandhiV: AT-101 induces apoptosis in CLL B cells andovercomes stromal cell-mediated Mcl-1 inductionand drug resistance. Blood (2009) ;113(1):149

6. Bannerji R, Byrd JC. Update on the biology of chronic lymphocytic leukemia. Curr Opin Oncol. 2000;12(1):22-29

7. Liu FT1, Giustiniani J, Farren T, Jia L, Bensussan A, Gribben JG, Agrawal SG: CD160 signaling mediates PI3K-dependent survival and growth signals in chronic lymphocytic leukemia. Blood. 2010 Apr 15;115(15):3079-88.

8. Jones B., Adams S., Miller G.T., Jesson M.I., Watanabe T., Wallner B.P. Hematopoietic stimulation by a dipeptidyl peptidase inhibitor reveals a novel regulatory mechanism and therapeutic treatment for blood cell deficiencies. Blood (2003) 102: 1641-1648

9. Binsky I, Haran M, Starlets D, et al. IL-8 secreted in a macrophage migration-inhibitory factor- and CD74dependent manner regulates B cell chronic lymphocytic leukemia survival. Proc Natl Acad Sci U S A. 2007;104(33):13408-13413.

10. Farren TW, Giustinian J, Lui FT, A. Tsitsikas D, Macey MG, Cavenagh JD, Oakervee HE, Taussig D, Newland AC, Calaminici M, Bensussan A, Jenner M, Gribben J and Agrawal SG. Differential and tumor-specific expression of CD160 in B-cell malignancies. Blood 2011 118:2174-2183

11. Terri L: Chronic Lymphocytic Leukaemia : prognostic factors and impact on treatment . Discov.Med(2011) ;11(57):115. 